\title{
LA MOTIVACIÓN Y SU USO EN LA RESOLUCIÓN DE PROBLEMAS
}

\section{THE MOTIVATION AND ITS USE IN TROUBLESHOOTING}

\author{
Mg. Paola Andrea Mina Gomez ${ }^{2}$ \\ Mg. Heberth Caicedo Saldaña ${ }^{3}$ \\ Unidad Central del Valle del Cauca, Colombia
}

\begin{abstract}
$1 \quad$ Articulo producto del trabajo articulado en la línea de investigación Pensamiento Crítico, de la facultad Ciencias de la Educación, de la Unidad Central del Valle del Cauca, Colombia, en el proyecto titulado: "Ambientes de aprendizaje y los estilos atributivos: Aplicación de un instrumento para identificar la motivación intrínseca (estilos atributivos) que manejan los estudiantes en la enseñanza de la licenciatura en lenguas extranjeras con énfasis en ingles dentro del contexto de logros académicos para emitir juicios reflexivos".

2

hcaicedo@uceva.edu.co

https://orcid.org/0000-0003-2609-3383

Docente investigadora de la Unidad Central del Valle (UCEVA) grupo ILA (Investigación en Lingüística Aplicada); Psicóloga de la Universidad de Manizales; Magister en Educación: Desarrollo Humano, de la Universidad San Buenaventura, Colombia; candidata a doctora en Ciencias de la Educación, Universidad de Cuauhtemóc, Aguas Calientes México.

3

pmina@uceva.edu.co

https://orcid.org/0000-0003-3523-183X
\end{abstract}

Docente investigador de la Unidad Central del Valle (UCEVA), Licenciado en Lenguas Modernas y Traducción de la Universidad del Quindío; Magister en Bilingüismo de la Universidad del Valle - Cali, Colombia; director del Grupo de investigación aprobado por Colciencias, ILA (Investigación en Lingüística Aplicada) de la facultad Ciencias de la Educación -UCEVA-.

\section{RESUMEN}

Al abordar la motivación como elemento fundamental en el proceso de enseñanzaaprendizaje de cualquiera sea el objeto de estudio desde el aprendiz, esta perspectiva se articula con la habilidad de resolución de problemas, la cual implica dinámicas cognitivas complejas, es así como este trabajo investigativo gira entorno a estos dos aspectos. Para abordar el campo motivacional y disposicional en relación con los componentes cognitivo y metacognitivo en la resolución de problemas, se utilizó un cuestionario con 38 afirmaciones, que hacen parte del test HCTAES y que busca evaluar la disposición de los aspectos del pensamiento crítico. El instrumento denominado: "Escala de conciencia" fue diseñado para evaluar una tendencia del individuo a "participar y a disfrutar de sus esfuerzos cognitivos". 


\section{PALABRAS CLAVE}

Pensamiento Crítico, Motivación, Resolución de problemas, Esfuerzo cognitivo, Escala de conciencia.

\section{ABSTRACT}

By addressing motivation as a fundamental element in the teaching-learning process of any object of study from the learner, this perspective is articulated with the ability to solve problems, which involves complex cognitive dynamics, is how this research work revolves around these two aspects. To address the motivational and dispositional field in relation to cognitive and metacognitive components in problem solving, a questionnaire was used with 38 affirmations, which are part of the HCTAES test and which seeks to evaluate the disposition of aspects of critical thinking. The instrument called: "Scale of consciousness" was designed to evaluate an individual's tendency to "participate and enjoy their cognitive efforts".

\section{INTRODUCCIÓN}

La motivación en sus diferentes acepciones ha sido un concepto muy recurrente de estudio para explicar la conducta humana, es un componente clave de los principios psicológicos centrados en el aprendiz (Beltrán, 2002; Santrock, 2006). Y aún más, la motivación es un aspecto crítico de la enseñanza y del aprendizaje, pues los estudiantes que no están motivados no realizan el esfuerzo necesario para aprender, mientras que los alumnos muy motivados están deseosos de ir a la universidad y se involucran en el proceso de aprendizaje.

Pero, la pregunta de interés por centrar el tema es ¿qué le es útil al sujeto para desvelar la realidad, para anticiparse, para formar sus proyectos existenciales y vivir originariamente hacia el futuro imaginándose en él? Como fenómeno psicológico la motivación no es comprendida como algo estático, sino, más bien, como un proceso que se encuentra compuesto por motivos, metas, percepción de la competencia y atribuciones, entre otros aspectos.

Si bien el acto de aprender implica una experiencia individual, es lógico pensar que ésta aparece como un registro inicial del deseo, de ser así, entonces seríamos sujetos acabados en las decisiones de experiencias y no se necesitaría del aprender como exterioridad. Por lo tanto, el entendimiento del aprendizaje requiere de elaborar el entendimiento de los aprendizajes, su importancia y desafíos que ellos aportan al mundo altamente cambiante, como seres inacabados (Zambrano, 2005; Artunduaga, 2008).

Es por ello, que la resolución de problemas se debe reconocer en los aprendizajes como productos de elaboraciones sociales en su conjunto, cuya gramática recae en los saberes como movilidad a la disposición en los procesos de aprender.

En la lógica tradicional de la clase, es decir en los ambientes de aula, el tiempo determina la sujeción del estudiante, ya que éste determina el tiempo en que el estudiante deberá alcanzar a comprender el todo, cuando el todo es mayor que las parte(Zambrano, 2005, p. 86).

\section{MOTIVACIÓN vs RESOLUCIÓN DE PROBLEMAS}

El aprendizaje en la vida académica y formal está determinado por variables cognitivomotivacionales, nos introduce de lleno en toda la compleja variedad de procesos y estrategias implicadas en el hecho de aprender.

Las comprensiones cognitivas modifican, desde luego, la conducta e influyen en la activación de motivos. Pero ¿se puede llegar a la conclusión de que un esquema de atribución causal define un motivo como señala Wiener? (McClelland, 1989; citado por Tapia, Sánchez, 1992). O que el motivo de logro es un sistema para evaluar 
el ego, como afirma Hechkausen (McClelland, 1989, p., 527). Mantener una actitud positiva que facilite superar el fracaso, y la actitud negativa evita fijarse objetivos de rendimiento, si ellos es posible, se fijan en objetivos irrealistas, muy altos o muy bajos, si tienen que fijarse algunos; rehúyen de la evaluación y atribuyen el éxito a la suerte y el fracaso a la falta de destreza. Así se ven sumidos en un ciclo de conducta defensiva que les impide hacerlo bien y satisfacerse con su rendimiento (McClelland, 1989).

En lo cognitivo se ubican la atención, la percepción y la memoria, como los principales procesos; la atención es condición básica para el funcionamiento de los otros procesos, implica además, la disposición neurológica para la recepción de estímulos (Reeve, 1995). Respecto a este punto, se configura la continuidad del estudio del pensamiento crítico, donde hay que considerar dos aspectos principales, el primero se refiere al carácter focal o selectivo, por medio del que se configura, y como segundo está el objeto que sobresale entre los demás estímulos y su carácter regulador o sostenido, como es la atención, la motivación, la memoria entre otros.

Con éstos elementos, el pensamiento crítico permite procesar y relaborar la información que recibe, dispone de una base de sustentación de sus propias creencias, posibilitando una actividad intelectual, tendiente a conseguir objetivos de manera eficaz, no tan sólo en el ámbito académico sino también en la vida diaria; en este tipo de pensamiento, se utiliza habilidades como: el razonamiento, la resolución de problemas, y la toma de decisiones.

Cabe resaltar también que ésta el pensamiento metacognitivo, el cual se refiere al grado de conciencia o conocimiento que los individuos poseen sobre su forma de pensar (procesos y eventos cognitivos). Los trabajos de Flavell (1981) que abordan los problemas implicados en la generalización y transferencia de lo aprendido, sirvieron para confirmar que los seres humanos son capaces de someter a estudio y análisis, los procesos que utilizan para conocer, aprender y resolver problemas; es decir, pueden tener conocimiento sobre sus propios procesos cognitivos, y además, controlarlos y regularlos (citado por Beltrán, 2002).

A partir de los trabajos de Flavell (1981), otros autores han realizado sus propias definiciones sobre la metacognición y sus componentes, es así como para Chadwick (1985) metacognición es la conciencia que una persona tiene acerca de sus procesos y estados cognitivos; para él, la metacognición se divide en sub-procesos, por ejemplo, meta-atención, referida a la conciencia que tiene la persona de los procesos que realiza para la captación de información; metamemoria, que hace alusión a los conocimientos que tiene un sujeto, de los procesos que le implican en el recuerdo de la información, como a la información que tiene almacenada en la memoria (contenidos de memoria), es decir, la conciencia de lo que conoce y de lo que desconoce (citado por Beltrán, 2002).

Para Costa (1984), la capacidad metacognitiva es un atributo del pensamiento humano, vinculada con la habilidad que tiene una persona para conocer lo que conoce, para planificar estrategias que le permiten procesar información; para tener conciencia de sus propios pensamientos durante el acto de solución de problemas; y para reflexionar y evaluar la productividad de su propio funcionamiento intelectual.

Por lo anterior, con el término metacognición se hace referencia al conocimiento acerca del propio conocimiento, asimismo, a los procesos cognitivos, afectivos, y a la habilidad deliberada para regularlos. Estos conocimientos y creencias acerca del pensamiento y los factores que lo afectan, son la pieza clave para regular las estrategias de conocimiento.

Es decir, la metacognición permite reflexionar sobre sí mismo, para descubrir sus propios 
procesos de pensamiento como objeto de examen. Con el término metacognición se hace referencia al conocimiento acerca del propio conocimiento, a procesos, estados cognitivos y afectivos, y a la habilidad consciente y deliberada para monitorear y regular dichos estados (Flavell, Miller, Miller, 2002).

Estos procesos cognitivos o habilidades de pensamiento, son finalmente, las herramientas de que dispone el alumno para procesar los contenidos y profundizar en el conocimiento. También es importante señalar, que la posibilidad de poner en práctica las habilidades de pensamiento, depende en gran parte del dominio específico en el que se despliegan, especialmente la motivación.

Por otro lado, el reconocimiento de los códigos propios de cada disciplina, posibilita el desempeño de ciertas destrezas y son la base frente al desafío de desarrollar habilidades de pensamiento.

\section{MÉTODO}

Para éste estudio se pretendió abordar el campo motivacional y disposicional en relación con los componentes cognitivo y metacognitivo en la resolución de problemas, se utilizó un cuestionario con 38 afirmaciones, que hacen parte del test HCTAES y que busca evaluar la disposición de los aspectos del pensamiento crítico, por lo que se proporcionan cuestionarios a los participantes.

El instrumento denominado: "Escala de conciencia" (Costa; Mc Rae, 1992) es una de las evaluaciones que se utiliza con el Factor Teoría de la Personalidad, los estudiantes utilizaron siete elementos que les permitieron hacer una descripción de su personalidad; de la misma manera el cuestionario fue diseñado para evaluar una tendencia del individuo a "participar y a disfrutar de sus esfuerzos cognitivos" (Cacciopo; Petty; Feinstein; Jarvis, 1996)
En este trabajo participaron 76 estudiantes del Programa de Licenciatura en Lenguas Extranjeras de la Uceva. La prueba fue resuelta de manera individual durante un tiempo de 60 minutos aproximadamente.

\section{ANÁLISIS DE RESULTADOS}

De acuerdo a los resultados, se puede identificar que la disposición (motivación vs resolución de conflictos) refleja en un $46 \%$ pero no muy significativo, que esta población estudiada permite procesar y relaborar la información que recibe. Es decir, que disponen de una base cognitiva que de sustentación de sus propias creencias, posibilitando una actividad intelectual, tendiente a conseguir objetivos de manera eficaz, no tan sólo en el ámbito académico sino también en la vida diaria; en este tipo de pensamiento, aunque resaltando que no es muy significativo, se utiliza habilidades como: el razonamiento, la resolución de problemas, y la toma de decisiones, habilitada desde una actitud mental intelectiva y afectiva suficiente para responder a los estímulos internos y externos.

Por otro lado, el esfuerzo cognitivo con un $47 \%$, el cual es atribuido al éxito o al fracaso, se ve reflejado desde una actitud negativa, a la no utilidad de la activación cognitiva. Teniendo este referente, es importante, ampliar y profundizar los elementos metacognición que sustentan la conciencia que una persona tiene acerca de sus procesos y estados cognitivos; con ello, lo que se pretende indicar es que la conciencia que tiene la persona de los procesos que realiza para la captación de información puede estar sujeta a una actitud positiva o negativa; referenciando que esta hace alusión a los conocimientos que tiene un sujeto, de los procesos que le implican en el recuerdo de la información, como a la información que tiene almacenada en la memoria (contenidos de memoria), es decir, la conciencia de lo que conoce y de lo que desconoce, por ello la importancia del rol docente en la orientación de ese esfuerzo cognitivo hacia una actitud 
positiva de éxito o fracaso, y no a una actitud negativa hacia el éxito o fracaso.

\section{CONCLUSIONES}

El interés por abordar el tema es que los problemas más graves de desmotivación en los alumnos surgen cuando éstos atribuyen sus propios errores a causas internas estables e incontrolables, por ejemplo, la capacidad, porque para ellos no existen soluciones. Caen, así, en un estado de apatía, de no saber quehacer, de indefensión. Sería preferible atribuir siempre los fracasos a causas externas que no dependen del control personal, como por ejemplo la suerte o la dificultad de las tareas. Lo positivo sería pensar que los problemas son solucionables, entrenables, sujetos a cambios, transitorios y modificables. Es por ello que, la existencia de unos estilos atribucionales de tipo adaptativo y desadaptativo deben ser abodados, en relación con el rendimiento académico. Hoy se sabe que los alumnos con un estilo adaptativo persiguen metas de aprendizaje, es decir, buscan el incremento de su propia competencia mediante la adquisición y dominio de nuevas habilidades y conocimientos, perfeccionando su ejecución en las tareas de aprendizaje y estudio. Sin embargo, aquellos otros alumnos que presentan un estilo motivacional y atribucional desadaptativo (dificultad en resolver problemas) están orientados hacia metas de resultado o rendimiento; en este caso, lo que pretenden es obtener un buen rendimiento centrándose en el producto o resultado final y no en el proceso de aprendizaje (Barca, Porto, otros, 2008).

Los logros académicos se pueden considerar como un modelo o estilo motivacional integrado de creencias, atribuciones y afectos/sentimientos que dirigen las intenciones de la conducta, es decir, de lo que los sujetos hacen o quieren hacer en contextos educativos de enseñanza/ aprendizaje, es por ellos que el interés está en determinar tanto las reacciones afectivas, cognitivas y conductuales del sujeto ante los resultados de éxito o fracaso, como la cantidad y calidad de sus actividades de aprendizaje y estudio.

\section{REFERENTES}

Artunduaga, M. (2008) Variables que influyen en el rendimiento académico en la universidad, departamento MIDE (Método de investigación y Diagnostico en Educación), Madrid - España. Julio. (Tesis doctoral). Complutense de Madrid.

- Barca, A., Porto, A., Vicente, F., Brenlla, J. C. y Morán, H. (2008). La interacción de estilos atribucionales y enfoques de aprendizaje como determinantes del rendimiento académico. Oviedo: Actas del $\mathrm{V}$ Congreso Internacional de Psicología y Educación: los retos del futuro.

- Beltrán, J. (2002). Cap. I Aprender. En Beltrán. Procesos, estrategias y técnicas de aprendizaje. Madrid: Síntesis.

- Costa, A. (1984). Mediating the metacognitive. Educational Leadership. 42(3), $57-62$

- Flavell, J.H, Miller, P.H., Miller, S.A. (2002). Cognitive development $\left(4^{\text {th }}\right.$ ed.). Upper Saddle River, Nj: Prentice Hall.

- McClelland, D. C. (1989). Estudio de la motivación humana. p. 527.

- Reeve, J. (1994). Motivación y emoción. McGrawHill/Interamericana de España, S. A.

- Santrock, J. (2006) Capitulo 13: motivación, enseñanza y aprendizaje. En John W. Santrock.(Eds.) Psicología de la Educación. (pp. 412-440). Segunda Edición. McGraw Hill/Interamericana editores, S.A. de C.V.

- Tapia, J. A.; Sánchez, J. C. (1992). Capítulo 3. Etilos atributivos y motivación: el cuestionario EAT. págs. 93-133 Tomado 
de: https://www.uam.es/gruposinv/meva/ publicaciones\%20jesus/libros_jesus/1992/ motivar_adolescencia_partes/cuestionario_ eat.pdf

- Zambrano, A. (2005) Didáctica, pedagogía y saber: aportes desde la ciencia de la educación. Cooperativa Editorial del magisterio, Bogotá, D.C., Colombia. 